\title{
Strates
}

STRATES Matériaux pour la recherche en sciences sociales

Hors-série | 2002

Parcours dans la recherche urbaine, Michel Rochefort, un géographe engagé

\section{Au Brésil : influences sur la géographie, engagement dans l'aménagement du territoire}

Jacques Brun et Catherine Paix

\section{(2) OpenEdition}

Journals

Édition électronique

URL : http://journals.openedition.org/strates/564

DOI : $10.4000 /$ strates.564

ISSN : $1777-5442$

Éditeur

Laboratoire Ladyss

Édition imprimée

Date de publication : 1 janvier 2002

ISSN : 0768-8067

Référence électronique

Jacques Brun et Catherine Paix, « Au Brésil : influences sur la géographie, engagement dans

l'aménagement du territoire », Strates [En ligne], Hors-série | 2002, mis en ligne le 18 mai 2005, consulté le 08 septembre 2020. URL : http://journals.openedition.org/strates/564 ; DOI : https:// doi.org/10.4000/strates.564

Ce document a été généré automatiquement le 8 septembre 2020.

Tous droits réservés 


\title{
Au Brésil : influences sur la géographie, engagement dans l'aménagement du territoire
}

\author{
Jacques Brun et Catherine Paix
}

1 Tu as joué un rôle très important au Brésil que ce soit sur le plan universitaire ou en matière d'aménagement du territoire. Comment expliques-tu que tes travaux aient eu très tôt un tel impact au Brésil et que tu y aies eu une telle influence intellectuelle?

2 Les relations interpersonnelles y ont été pour beaucoup. Les clivages au sein de la géographie brésilienne également. Cela a commencé en 1956, au moment du fameux Congrès international de Géographie à Rio de Janeiro, qui a été le premier Congrès international de l'UGI dans un pays en développement. J'étais alors jeune assistant à l'université de Strasbourg, en train de remuer un peu la géographie brésilienne, et cela m'a fait connaître. J'avais connu - par l'intermédiaire de Regina, mon épouse, qui était brésilienne et géographe -, un groupe de jeunes géographes qui en avaient assez de la géographie classique qu'on leur avait apprise, par le biais notamment des Français, et du mandarinat brésilien, et qui voulaient que je fasse des conférences, au département de Géographie de São Paulo. Et lorsque le directeur leur a refusé en disant «Les géographes français en qui j'ai toute confiance estiment que Michel Rochefort a des idées dangereuses ", ils se sont rapprochés de moi. C'est un peu comme cela que j'ai commencé à être invité au Brésil, d'abord un an à Recife, pour lancer un programme d'enseignement de géographie humaine appliquée, puis en 1961, au Conseil national de Géographie, où j'ai travaillé pendant six mois, pour réformer l'ensemble des recherches de géographie au Brésil. Le Conseil national de Géographie du Brésil était encore organisé en sections régionales, c'est-à-dire qu'il y avait un regroupement des géographes travaillant sur chaque région, l'Amazonie, le Nord-Est, le Sud-Est, etc., et ils voulaient créer une structure parallèle à base thématique. Je me suis donc remis à la géographie dans de nombreux domaines, puisque j'ai proposé une section de géographie urbaine, une de géographie agraire, etc., et, à partir de là, j'ai essayé de mobiliser des groupes de géographes. J'organisais des séminaires de discussion pour 
dégager des thématiques dans les différents champs de la géographie, particulièrement en géographie urbaine, et finalement tout cela a fait souche, et des tas de gens sont venus travailler avec moi. Puis, à partir de 1966, il y a une géographe brésilienne qui a joué un rôle important dans ma collaboration avec le Brésil, c'est Maria Adelia De Souza, qui avait d'abord travaillé avec Pierre Monbeig, mais qui l'avait quitté pour faire sa thèse sous ma direction. Lorsqu'elle est revenue à São Paulo, elle a été confrontée au même ostracisme de la part des mandarins encore en place, parce qu'elle avait travaillé avec moi, mais elle a été assez forte pour s'imposer dans le domaine de la géographie appliquée, elle est devenue responsable au secrétariat d'État chargé de la décentralisation industrielle à São Paulo, et elle m'a fait venir au Brésil. Ma collaboration dans le domaine de l'aménagement au Brésil a donc commencé là, par le biais d'une de mes anciennes élèves. Puis, progressivement les vieux mandarins sont partis, Maria Adelia est devenue une personne très reconnue, - elle a même reçu le prix de la Francophonie pour ce qu'elle avait fait - et j'ai travaillé avec les services d'aménagement du Brésil, au secrétariat d'État à l'aménagement du territoire de São Paulo, où elle était l'équivalent de sous-secrétaire d'État, mais aussi à la Commission nationale de politique urbaine de Brasilia et au ministère du Plan où elle était aussi. Cela a duré de 1966 à 1969, puis il y a eu une interruption entre 1969 et 1973, à cause de la dictature "dure», durant laquelle je n'ai pas pu retourner au Brésil. Ma collaboration avec la Commission nationale de politique urbaine a repris à partir de 1974, au même moment où les jeunes géographes qui m'avaient écouté quelques années plus tôt ont commencé à avoir des postes importants. J'ai alors été invité à faire des conférences un peu partout au Brésil ; de Porto Alegre à Florianapolis en passant par Presidente Prudente, Manaus, Recife, Belem, Salvador, Brasilia, etc.

3 Quelle a été la nature de ton intervention dans l'aménagement du territoire au Brésil ? Sur quels problèmes ont porté tes travaux?

4 J'intervenais, surtout après 1968, en tant qu'expert international envoyé par le ministère des Affaires Étrangères français auprès des services d'aménagement du territoire. J'ai fait quinze missions de coopération technique, et, à partir de 1974, quand les dirigeants brésiliens ont marqué leur volonté d'engager le débat sur les problèmes sociaux, je suis entré dans les services d'aménagement du territoire, au niveau fédéral et à celui de l'État de São Paulo, où j'ai participé à l'élaboration de plusieurs programmes d'aménagement, qui ont fait l'objet de différents rapports, en particulier sur les villes moyennes, la déconcentration industrielle de l'État de São Paulo, et la réforme administrative.

5 Quelles ont été les répercussions de ces études? Ont-elles contribué à des transformations notables dans la dynamique urbaine au Brésil ?

6 Indirectement, cela a eu des répercussions importantes, parce que les analyses et les propositions que nous faisions rejoignaient les intérêts des acteurs économiques euxmêmes. Cela a été très clair à São Paulo, où les investisseurs et les industriels avaient bien conscience des blocages auxquels conduisait la croissance du grand São Paulo et des effets négatifs de la concentration urbaine en termes de coûts et de danger social. Ils voyaient donc tout l'intérêt que pouvaient présenter la récupération des villes moyennes et la déconcentration industrielle. Celle-ci se serait donc faite de toutes façons. Mais nos études ont quand même contribué à orienter les investisseurs vers telle ville plutôt que telle autre, car il y avait ce qui s'appelait « un balcon des projets ", où certains d'entre eux venaient discuter avec Maria Adelia De Souza pour savoir où ils 
devaient s'implanter. Cette marge de manœuvre dont on disposait a donc sans doute servi les intérêts capitalistes, ce qui est un peu inévitable lorsque l'on fait de l'aménagement, mais en même temps cela a eu un certain effet, certes modeste, sur la diminution de la croissance de São Paulo et la réanimation d'un certain nombre de villes moyennes qui sont devenues des villes importantes.

7 Après ton expérience française, qu'est ce qui t'a incité durant cette période à t'engager dans l'aménagement au Brésil? Dans quelle mesure tes réflexions sur le Tiers-Monde et ta connaissance de la réalité brésilienne ont-elles changé ta vision des choses?

8 J'y ai cru davantage, parce que j'ai pensé qu'il y avait une plus grande marge de manœuvre. Au Brésil, il y avait beaucoup moins de conflits que ceux que je vivais en France, et je n'étais pas dans la même position par rapport au politique. J'y ai aussi toujours été plus en accord avec moi-même parce que tous mes amis brésiliens, tous ceux avec lesquels j'ai collaboré, ont toujours été du même côté que moi sur le plan idéologique. À partir de 1966-1967, j'ai pris plus nettement conscience de la réalité urbaine brésilienne, ce qui m'a conduit à remettre en cause ce que j'avais fait antérieurement sur la France. J'ai aussi été très marqué par le grand voyage que j'ai fait à travers les villes d'Inde en 1968, par les recherches menées sur l'impérialisme dans le cadre du laboratoire de Géographie humaine à partir de 1970, et par mes contacts avec l'Afrique dans le cadre de mes fonctions à l'ORSTOM à partir de 1973. Tout cela m'a poussé à réfléchir sur l'impact de la domination dans l'organisation de l'espace dans les pays en développement, à mieux cerner les différences, la spécificité des formes de développement urbain dans ces pays, et finalement à en conclure qu'étant donné le poids de l'héritage colonial et de la domination sur la distribution des villes au Brésil, il n'y avait pas beaucoup de possibilités d'aménagement. À l'époque je me suis d'ailleurs opposé fortement à ceux qui, comme en Côte d'Ivoire par exemple, croyaient au miracle ivoirien et parlaient de modèles de développement régional, en soutenant qu'étant donné la structure de l'économie, le système urbain resterait déséquilibré, entre un port omnipotent et des villes trop petites à l'intérieur. Mes recherches m'ont donc amené à voir ce qu'il y avait d'inéluctable dans l'organisation urbaine de ces pays. Mais elles m'ont conduit aussi à mieux cerner ce qu'il était possible de faire en matière d'aménagement pour corriger un peu les choses.

9 Tu viens de dire que tu pensais qu'il y avait au Brésil une plus grande marge de manceuvre, qu'en a-t-il été de tes relations avec les autorités politiques et de la réception de vos travaux dans le contexte politique du Brésil de l'époque ? À quelles difficultés t'es-tu heurté à ce niveau?

10 Je n'ai pas eu au Brésil les mêmes responsabilités qu'en France au moment des métropoles d'équilibre, et mes relations avec les autorités politiques ne se sont pas posées dans les mêmes termes, car je n'intervenais qu'en tant qu'expert international. Ceux qui ont fait appel à moi partageaient mes idées et pensaient comme moi qu'il y avait au niveau de l'aménagement du territoire une certaine marge de manœuvre pour essayer de corriger les déséquilibres existants et faire bouger les choses dans le sens du progrès social. Cela s'est donc heurté à une classe politique qui n'avait pas une très grande envie de poser les problèmes de progrès social, et on a fait ou non appel à moi, en fonction de ce que je pouvais représenter, sur le plan idéologique, comme conception globale de la société. Mais d'une façon générale, je n'ai pas rencontré trop de difficultés pour travailler. À l'époque de la dictature, tout était très surveillé et il fallait faire attention à ce que l'on disait, ne pas prononcer le mot de sousdéveloppement par exemple, au risque d'être remis dans l'avion immédiatement, mais 
j'étais protégé par mon statut d'expert international, et à ce moment-là, mon rôle a surtout consisté à engager des débats avec des chercheurs qui n'étaient pas dans la clandestinité, ni impliqués dans la recherche appliquée. On a donc travaillé en grande partie à la marge, et de ce fait il n'y avait pas trop de surveillance sur ce que l'on faisait. Ce travail a d'ailleurs été réutilisé à São Paulo. Puis, entre 1974 et 1978, quand il y a eu une ouverture de la dictature, et que je suis entré dans les services d'aménagement, on nous a laissés mener nos travaux librement.

11 Ceci dit, la relation avec le politique n'en est pas moins complexe et ambiguë. À São Paulo, le secrétariat d'État à l'aménagement avait été confié à un économiste, qui était un universitaire et qui avait dans son bureau une représentation du très beau poème d'Eluard sur la liberté : «Par le pouvoir d'un mot je recommence ma vie, je suis né pour te connaître, pour te nommer, Liberté ", et il nous laissait faire, proposer des programmes, expliquer ce que l'on avait à dire, mais il nous laissait faire aussi parce qu'en réalité, ce que l'on faisait n'était pas considéré comme important, et n'impliquait pas de grandes dépenses, puisque nos études visaient à convaincre les investisseurs, qui étaient pratiquement déjà convaincus -, qu'il fallait faire autre chose.

12 Au niveau fédéral cela a été plus compliqué, car il fallait convaincre les autorités politiques réelles, qui étaient les militaires, et là aussi le rôle des personnes qui ont été nos interlocuteurs a été important. Entre 1974 et 1978, le ministre du Plan était un économiste qui avait été auparavant responsable des premiers services d'aménagement des villes, le SERFAU. Il était intéressé par tous les problèmes que nous posions, et je crois qu'il a réussi à convaincre les autorités politiques réelles, qui étaient les militaires, en leur montrant que la grande ville représentait un réel danger, et qu'il fallait renforcer les villes moyennes pour réduire la pression sur les grandes villes. Il nous a donc fait travailler sur l'aménagement du système urbain brésilien, et on a eu les coudées franches pendant quatre ans pour monter un programme d'appui aux villes moyennes.

Mais il faut voir que cela correspondait aussi à la politique brésilienne d'intériorisation du développement, dont Brasilia était le symbole.

14 Dans quelle mesure vos travaux ont-ils été suivis de politiques effectives? Quelles en ont été la portée politique et les limites?

15 Nos propositions n'ont pas toujours été acceptées. Au départ, nous avons été très modestes. Nous sommes d'abord partis de l'idée qu'on ne pouvait pas changer le système urbain, et que la première chose à faire était de diminuer les contradictions internes aux grandes villes littorales, ce qui nous a conduit à proposer la création d'aires métropolitaines et d'une loi sur l'aménagement des aires métropolitaines. Le projet visait à donner un certain pouvoir à ces aires métropolitaines, c'est-à-dire à la ville-mère, plus les 10,15 ou 20 communes des environs, et à les doter d'une institution pour gérer les problèmes à l'échelle de l'agglomération, sans bien sûr mettre à mal le pouvoir de chaque municipe mais en essayant quand même de créer un échelon intermédiaire qui n'existait pas au Brésil entre l'État et la ville. Ce projet a été immédiatement barré, car cela touchait directement aux intérêts et à la bourgeoisie de chaque municipe, mais cela n'est pas venu des militaires, et la Commission a pu continuer ses études sur les villes moyennes. On a effectivement choisi 37 villes qui nous paraissaient être les plus importantes, on a essayé de dégager les moyens d'action possibles, en fonction de l'économie de chaque région, et on a sorti un programme. Sa réalisation coûtait cependant trop cher, et le gouvernement fédéral a du faire appel à la 
BIRD, qui n'a accepté de financer que dix villes parmi les trente sept. Ce premier programme d'appui aux villes moyennes continue, et il est certain que les dix villes en question ont changé parce qu'il y a eu un investissement significatif. Mais cela a constitué dix exemples intéressants de villes qui se sont mieux développées que d'autres sans que ce développement ait un quelconque effet sur l'ensemble du système urbain brésilien.

16 Après 1978, la question du développement des villes moyennes est devenue un problème beaucoup plus politique. La Commission nationale de politique urbaine a été supprimée, avec l'arrivée au ministère du Plan d'un économiste représentant les intérêts américains au Brésil, qui défendait l'idée que les grandes villes se développaient plus que les autres parce qu'elles généraient la meilleure croissance $d u$ PIB. Le ministère de l'Intérieur de l'époque avait cependant très bien compris l'intérêt qu'il pouvait y avoir sur une plan strictement politique à financer certaines villes, puisqu'en dehors des métropoles, les villes moyennes avaient des pouvoirs municipaux élus -, et le gouvernement a effectivement choisi un certain nombre de villes, - 120 environ -, dont la majorité politique lui convenait, et auxquelles il a accordé de petits financements au nom d'un programme d'appui aux villes moyennes.

Voilà la perversion politique de cette histoire. Quant à moi, je n'ai plus participé à la politique urbaine à partir de ce moment là. Ceci étant, comme il y avait eu un accord avec la Banque mondiale, le programme d'appui aux dix villes - devenues onze par la suite - a perduré, et bien que cela n'ai pas radicalement changé les choses, il n'en est pas moins vrai qu'aujourd'hui on peut y constater une certaine amélioration des conditions de vie.

\section{AUTEURS}

JACQUES BRUN

Professeur à l'université de Paris I, Ladyss

CATHERINE PAIX

Ladyss, Cnrs 